\section{Gout (1st Edition)}

Nicola Dalbeth, Lisa Stamp, and Tony Merriman, eds. Oxford: Oxford University Press, 2016, 128 pages, $\$ 34.50$ US

Gout is a well-written and concise reference for clinicians seeking an update on gout. It neatly summarizes both the basic scientific aspects of gout and hyperuricemia as well as current best practices in the clinical management of patients with gout. The key points at the beginning of each chapter outline the most important concepts while the references at the end include all the landmark studies on each topic.

The first few chapters provide a succinct overview of the pathogenesis, epidemiology, and genetics behind hyperuricemia and gout. Insights from the recent studies have been collated in a format that would be easily understood by clinicians.

The next 3 chapters focus on the clinical, laboratory, and radiographic features of gout. The chapter on imaging is especially well written, with a table that compares and contrasts how various features of gout can be detected by different modalities. Unfortunately, only 4 color plates with high-resolution images are included on the inside covers, while the rest are grainy black and white images that have not done justice to the actual images seen in clinical practice.
The next 4 chapters describe current principles in the management of gout, from acute flares to chronic urate-lowering therapies. The authors should be applauded for devoting an entire chapter to the management of comorbid conditions seen in patients with gout because the interplay between the management of these conditions and that of gout is a common challenge for both patients and clinicians.

The last 2 chapters deal with research tools and the future direction of gout management. The authors should be praised again for writing these chapters in a way that enlightens clinicians without diving too heavily into minutia.

This book would be a welcome addition to the library of primary care and emergency physicians as well as internists and rheumatologists. Its readability also means that trainees should be able to comprehend the context without difficulties.

GREGORY CHOY, MD, FRCPC, Rheumatology Division, Sunnybrook Health Sciences Centre. Address correspondence to Dr. G. Choy, Rheumatology Division, Sunnybrook Health Sciences Centre, 2075 Bayview Ave., Toronto, Ontario M4N 3M5, Canada.

E-mail: gregory.choy@sunnybrook.ca,gregory_choy@yahoo.ca

J Rheumatol 2017;44:1; doi:10.3899/jrheum.161294 